\title{
Extra-Hepatic Biliary Obstruction - A Cross-Sectional Study to Assess the Various Benign and Malignant Causes for Obstructive Jaundice among Patients Attending Trivandrum Medical College
}

\author{
Swasthik K.S. ${ }^{1}$, Preetha Sundaresan ${ }^{2}$, Varun Vijayan ${ }^{3}$ \\ 1,2,3 Department of General Surgery, Thiruvananthapuram Government Medical College, \\ Kerala University of Health Sciences, Kerala, India.
}

\section{ABSTRACT}

\section{BACKGROUND}

Extra-hepatic biliary tract obstruction can be due to a number of conditions. Most causes are due to stones in the common biliary duct or due to malignant obstruction. Malignant causes include carcinoma head of pancreas, periampullary carcinoma and cholangiocarcinoma. Besides calculus and malignant obstruction, benign and malignant strictures can also cause obstruction of extra hepatic biliary ducts. The objectives of the study were to describe the clinical profile of patients presenting with extra-hepatic biliary obstruction and to assess the known aetiological factors.

\section{METHODS}

The research was designed as a hospital based cross-sectional study in the general surgery wards of our institution from 2016 to 2017. All individuals who were diagnosed to have extra-hepatic biliary obstruction by imaging were included in the study.

\section{RESULTS}

Among the 66 cases studied, majority were due to malignancies and gall stones. The malignancies included carcinoma head of pancreas, periampullary carcinoma, cholangiocarcinoma and Klatskin's tumour. Miscellaneous causes were bile duct stricture, cholelithiasis with biliary sludge and annular pancreas. For the malignant cases, Whipple surgery and its pylorus-preserving variant were the most common surgical procedures carried out. Biliary bypass procedures were also carried out in some patients. For patients with common bile duct (CBD) stones, cholecystectomy, choledocholithotomy and choledochojejunostomy were done.

\section{CONCLUSIONS}

Malignant causes of jaundice are more common than benign causes. Secondary stones are the commonest cause of non-malignant biliary obstruction. Jaundice is more severe and associated with pruritis and more intolerable and persistent in malignancy. Surgical bypass procedures give good palliation for obstructive jaundice.

\section{KEY WORDS}

Bile Ducts, Choledocholithiasis, Cholestasis, Extra Hepatic, Pancreatic Cancer, Periampullary Cancer
Corresponding Author:

Preetha Sundaresan,

TC 2/749, "Revathi",

MCRA 124, Chalakuzhy Road,

Medical College,

Thiruvananthapuram - 695011,

Kerala, India.

E-mail: arulakam69@gmail.com

DOI: 10.14260/jemds/2021/129

How to Cite This Article:

Swasthik K.S., Sundaresan P, Vijayan V. Extra-hepatic biliary obstruction - a crosssectional study to assess the various benign and malignant causes for obstructive jaundice among patients attending trivandrum medical college. J Evolution Med Dent Sci 2021;10(09):600-603, DOI: 10.14260/jemds/2021/129

Submission 18-08-2020,

Peer Review 06-01-2021,

Acceptance 12-01-2021,

Published 01-03-2021.

Copyright (C) 2021 JEMDS. This is an open access article distributed under Creative Commons Attribution License [Attribution 4.0 International (CC BY 4.0)] 


\section{BACKGROUND}

Biliary as well as duodenal obstruction is a common complication in patients with pancreatico-biliary malignancies. Obstructive jaundice with accompanying duodenal obstruction is commonly caused by periampullary malignancy, pancreatic head carcinoma, cholangiocarcinoma, malignant duodenal tumour or sometimes by lymph node metastasis. The other major share of obstructive jaundice is contributed by stones occluding the common bile duct. Biliary and duodenal obstruction leads to cholestasis with subsequent hepatic insufficiency.

In most cases, diagnosis of a case of obstructive jaundice is based on clinical, biochemical, imaging and endoscopic techniques. In obstructive jaundice, there is often elevation in serum alkaline phosphatase, gamma-glutamyl transpeptidase, and serum bilirubin levels. When there is no prior biliary intervention, patients commonly present with jaundice when their serum bilirubin level goes above $3 \mathrm{mg} / \mathrm{dL}$. But in some instances diagnosis may be difficult and exploratory laparotomy and even intra-operative radiological techniques may be needed for proper management. CA 19 - 9 is the most frequently studied biomarker in screening for pancreatic cancer and has a reported sensitivity of $79 \%$ and specificity of $82 \%{ }^{1}$ However, CA 19 - 9 has the drawback of being frequently elevated in other gastrointestinal cancers (cholangiocarcinoma, gastric cancer, colorectal cancer, oesophageal cancer and hepatocellular cancer) and even benign processes (acute cholangitis and pancreatitis).2,3

Multiple imaging modalities have been used to diagnose and evaluate the extent of obstructive jaundice. Exclusive diagnostic modalities include trans-abdominal ultrasound, helical computed tomography (CT) scan, and magnetic resonance imaging (MRI) or magnetic resonance cholangiopancreatography (MRCP). Newer modalities that offer options for both diagnosis and therapeutic interventions include endoscopic ultrasound (EUS), endoscopic retrograde cholangiopancreatography (ERCP), and percutaneous transhepatic cholangiopancreatography (PTC).

Patients with advanced obstructive jaundice are often critically ill. Subsequently, proper pre-operative preparation, appropriate surgery and careful post-operative management are essential for successful outcome of the cases and prevention of serious complications. Exploration of the disease presentation and the pathophysiology can give a proper idea about these patients and the disease process. With this background, we tried to study the cases of obstructive jaundice managed in the surgical wards of our institution. Government Medical College Trivandrum is a tertiary level teaching hospital in the southern part of Kerala and caters to the specialised health needs of a large number of population. The primary objective of the study was to describe the clinical profile of patients presenting with extra-hepatic biliary obstruction among the in-patients of the Department of General Surgery in our hospital. The secondary objectives were to assess the proportions of known aetiological factors associated and also the methods of treatment of extra-hepatic jaundice.

\section{METHODS}

The research was designed as a hospital based cross-sectional study. All individuals with extra-hepatic jaundice as shown by imaging in our surgical wards were taken as the study population. Patients less than 13 years were excluded for homogeneity. Census type sampling was employed for recruiting cases. The study was carried out for a period of 15 months, from May 2016 to September 2017. Prior to starting the study, clearance was obtained from institutional review board and ethics committee.

Informed consent was obtained from all patients and recorded. The patients were enquired in detail about their symptomatology and the responses were entered into a structured questionnaire. Also, relevant information on the patients' examination findings and laboratory investigations was entered into the structured proforma. The patients were followed up till their treatment and till discharge. All relevant treatment details were recorded in the proforma.

Study variables included name, age, gender, history of previous surgeries, laboratory values, contrast-enhanced computed tomography (CECT) abdomen findings, intraoperative findings and histopathological findings. The data was entered into Microsoft Excel sheets and subsequently used for analysis. All statistical analysis was done with 'Epi Info' software by CDC, Atlanta. All values were given as means and percentages as relevant. It was decided to set statistical significance at a $\mathrm{P}$ value lower than 0.05 , wherever relevant.

\section{RESULTS}

At the end of the study, we had 66 patients to analyse. Out of 66 patients, 33 were between the age group of $41-60$ years of age. Youngest patient was 27 years old and the oldest was 78 years of age. Male to female ratio was almost equal, with slight preponderance in males.

Jaundice was the main symptom seen among $55 \%$ of patients followed by pain in abdomen and loss of weight. $55 \%$ of the patients had associated history of smoking, ethanol consumption or both. $55 \%$ patients presented with icterus. 45 $\%$ patients had either palpable hepatomegaly or palpable gall bladder. $12 \%$ of them had only hepatomegaly.

$50 \%$ of the patients were having anaemia with haemoglobin lower than $11 \mathrm{gm} \% .14 \%$ patients had total bilirubin less than $2.5 \mathrm{mg} / \mathrm{dl}$ while $52 \%$ had bilirubin more than $5 \mathrm{mg} / \mathrm{dl}$. Among the 66 patients, liver enzymes, namely serum glutamic-oxaloacetic transaminase (SGOT) and serum glutamic-oxaloacetic transaminase (SGPT) were elevated in 65 $\%$ and $60 \%$ respectively. Alkaline phosphatase (ALP) was normal among $33 \%$ and elevated more than 600 in $15 \%$. Serum total protein was less than $6 \mathrm{mg} / \mathrm{dl}$ and serum albumin less than $3 \mathrm{mg} / \mathrm{dl}$ in $26 \%$ and $29 \%$ of the patients respectively. Serum CA 19 - 9 was found to be elevated among $49 \%$ of the patients and elevated to more than 10 times of normal in $18 \%$.

$15 \%$ of the patients had lesions in ampulla or periampullary area in upper gastrointestinal (GI) endoscopy. On radiological imaging, pancreatic malignancy topped the chart followed by periampullary malignancy and cholelithiasis with choledocholithiasis. Among the patients diagnosed with extrahepatic biliary obstruction, $67 \%$ patients were diagnosed with malignant causes. Pancreatic malignancy constituted the highest with $29 \%$ patients, followed by periampullary and cholangiocarcinoma constituting $26 \%$ and $11 \%$ respectively. Among the benign diseases, cholelithiasis with 
choledocholithiasis constituted the highest with $12 \%$ of patients. Congenital cause namely annular pancreas constituted 1 among 66 cases.

$77 \%$ of patients were treated with surgical procedure and the $11 \%$ were treated medically with drugs and endoscopic stenting. Among the patients treated with surgical procedure, $45 \%$ underwent curative surgical procedure and rest of them underwent palliative procedure. Choledochojejunostomy with gastrojejunostomy were done in $27 \%$ of the patients. Classic Whipple procedure and its pylorus-preserving pancreaticoduodenectomy variant (PPPD) were done in $26 \%$ patients. Bile duct stenting was done in $17 \%$ patients.

\begin{tabular}{|ccc|}
\hline Procedure & Frequency & Percent (\%) \\
Cholecystectomy & 4 & 6.1 \\
Cholecystectomy + CBD exploration & 1 & 1.5 \\
Cholecystectomy + CBD exploration + & 4 & 6.1 \\
Choledochoduodenostomy & 1 & 1.5 \\
Classic Whipple & 16 & 24.2 \\
PPPD & 18 & 27.3 \\
Cholecystojejunostomy + gastro-jejunostomy & 3 & 4.5 \\
Choledocho-jejunostomy & 1 & 1.5 \\
Roux-en-Y loop surgery & 1 & 1.5 \\
Frey's + cholecystectomy + choledocho & 11 & 16.7 \\
Jejunostomy & 6 & 9.1 \\
Stenting & 66 & 100 \\
\hline Others & \\
\hline Total & \\
\hline Table 1. Frequency of Surgical Procedures & \\
\hline
\end{tabular}

\begin{tabular}{|ccc|}
\hline Diagnosis & Frequency & Percent \% \\
Periampullary & 17 & 25.8 \\
HOP / CA pancreas & 19 & 28.8 \\
Cholangiocarcinoma & 7 & 10.6 \\
GB ca & 1 & 1.5 \\
CBD stricture & 5 & 7.6 \\
CBD stone & 2 & 3 \\
GB stone / polyp & 4 & 6.1 \\
GB and CBD stone & 8 & 12.1 \\
Congenital & 1 & 1.5 \\
Others & 2 & 3 \\
Total & 66 & 100 \\
\hline Table 2. Frequency of Various Diagnosis \\
\hline \multicolumn{2}{|}{} \\
\hline
\end{tabular}

\section{DISCUSSION}

Malignant biliary tract obstruction (MBTO) can arise from pancreatic, gallbladder, duodenal, ampullary and hepatic cancers; metastatic cancers; or malignant lymphadenopathy. Symptoms of obstructive jaundice specifically pruritus can adversely impact quality of life and subsequently promote hepatic dysfunction. Development of MBTO in malignancies may lead to poor outcomes including cholangitis, delay in proper treatment (including chemotherapy or surgery) and also increased mortality.

In spite of the advances in surgery and chemotherapy, pancreatic ductal adenocarcinoma has a dismal five-year survival rate of around $6 \%$, and biliary obstruction is known to correlate with decreased survival rates. ${ }^{4}$ Around two-thirds of patients have some degree of biliary obstruction when they get diagnosed with pancreatic cancer. ${ }^{5}$ In fact, jaundice is often the presenting symptom in up to $90 \%$ of pancreatic cancers involving the head of the gland. 6 This manifests as scleral icterus or yellow appearance of the skin. Pruritus, pale stools, dark urine, right upper quadrant discomfort and nausea are the other common symptoms. Other nonspecific symptoms pointing towards malignancy might include weight loss, anorexia and also night sweats.

Specific symptoms indicative of pancreatic cancer include dull epigastric pain with radiation to the back (caused by pancreatic duct obstruction), palpable gall bladder, generalised weakness and dyspepsia (due to gastric outlet obstruction as well as malabsorption from reduced pancreatic enzymes), early satiety, new onset diabetes mellitus or episodes of acute pancreatitis. ${ }^{7}$ Symptoms suggestive of cholangiocarcinoma include abdominal pain (mostly right upper quadrant), pruritus, weight loss and fatigue. ${ }^{8}$ Perihilar and distal common bile duct lesions tend to present predominantly with jaundice. ${ }^{9}$

Bilioduodenal strictures are classified according to the location as well as relative timing of duodenal obstruction. Type I strictures have duodenal obstruction located proximal to the papilla. ${ }^{10}$ Type II and III bilioduodenal strictures have the location of duodenal obstruction situated at or distal to the duodenal papilla, respectively. Bilioduodenal stenosis is classified according to the degree of duodenal obstruction as well as the relative timing of obstruction. In case of mild degree of duodenal obstruction, biliary cannulation is usually possible using a slim duodenoscope or after gentle dilatation of the stenosis. However, duodenal obstruction of a significant degree necessitates prior placement of an enteral stent.

One of the most common causes of benign biliary stricture is iatrogenic (post-surgical anastomotic stricture). Other reasons for strictures include post-traumatic, congenital, inflammatory, ischemic and idiopathic. In patients who have undergone biliary reconstruction after surgery for malignancy, it is often challenging to differentiate between anastomotic strictures and tumour recurrence.

Biliary intervention procedures play an important role in the management of patients with benign as well as malignant obstructive jaundice. The procedures include a variety of interventions including percutaneous transhepatic biliary drainage (PTBD) with or without stent placement, percutaneous transhepatic cholangiography (PTC), bile duct stone extraction and bile duct biopsy. In case of malignant bile duct obstruction, these interventions can be used for palliation of symptoms including anorexia and pruritus. ${ }^{11}$ It can also facilitate to prevent toxicity in some chemotherapy regimens by allowing total bilirubin levels to drop to acceptable levels. ${ }^{12}$ Biliary drainage is also performed routinely pre-operatively by some surgeons but its use in this context is controversial. ${ }^{13}$

Biliary stents have gained prominence in achieving biliary decompression over the past few decades. Recent studies show increased role for self-expandable metal stents (SEMS) compared to plastic stents. ${ }^{14}$ Endoscopic biliary stent placement is technically successful in over most of the attempted cases. ${ }^{15}$ For this reason, endoscopic retrograde cholangio-Pancreaticography (ERCP) together with biliary stent placement has now come to be accepted as the optimum standard of care in situations where biliary decompression is warranted. Another option is Endoscopic ultrasound-guided biliary drainage (EUS-BD) which can be achieved by multiple techniques including EUS-guided rendezvous, EUS-guided choledochoduodenostomy (EUS-CDS), and EUS-guided hepatic gastrostomy (EUS-HGS). ${ }^{16}$ Also, surgical biliary bypass can be used as an appropriate treatment for patients in whom life expectancy exceeds six months. ${ }^{17}$

Regarding surgical management, only $15 \%$ to $20 \%$ of patients are considered surgically resectable at diagnosis. ${ }^{18}$ Classically, the curative resection for cancers in the head of the pancreas was performed through pancreatoduodenectomy (Whipple procedure), which is a major surgical procedure 
involving removal of the pancreatic head, duodenum, gallbladder, common bile duct, gastric antrum, and the first 15 $\mathrm{cm}$ of jejunum. This procedure was later modified into a version that retains the stomach in situ, called pyloruspreserving pancreatoduodenectomy (PPPD). Meta-analyses comparing the techniques have found the two techniques to be equivalent in morbidity, mortality and overall survival. ${ }^{19}$ Operating time and blood loss are often significantly less in the pylorus-preserving pancreatoduodenectomy variant. Palliative surgical drainage is often prompted by obstructive jaundice, which is often accompanied by gastric outlet obstruction that precludes endoscopic biliary drainage. Cholecystojejunostomy and cholecystoenterostomy are the commonly performed procedures, though hepaticojejunostomy is shown to be superior to both. ${ }^{20}$

\section{CONCLUSIONS}

Extra-hepatic biliary obstruction is a challenging disease from both diagnostic and management points of view. Multiple diagnostic and therapeutic modalities are available and almost all patients can have their obstruction relieved through curative or palliative treatment options. Endoscopic, radiologic and surgical approaches remain as complementary techniques and not as competitive modalities. Choice of procedure should ideally be selected based on the location and type of biliary obstruction, local expertise, patient's performance status and patient's preference.

Data sharing statement provided by the authors is available with the full text of this article at jemds.com.

Financial or other competing interests: None.

Disclosure forms provided by the authors are available with the full text of this article at jemds.com.

\section{REFERENCES}

[1] Goonnetilleke KS, Siriwardena AK. Systematic review of carbohydrate antigen (CA 19-9) as a biochemical marker in the diagnosis of pancreatic cancer. Eur J Surg Oncol 2007;33(3):266-70.

[2] Steinberg W. The clinical utility of the CA 19-9 tumor associate antigen. Am J Gastroenterol 1990;85(4):350-55.

[3] Mann DV, Edwards R, Ho S, et al. Elevated tumour marker CA19-9: clinical interpretation and influence of obstructive jaundice. Eur J Surg Oncol 2000;26(5):474-9.

[4] Mahendraraj K, Chamberlain RS. Trends in incidence, survival and management of uveal melanoma: a population-based study of 7,516 patients from the surveillance, epidemiology, and end results (SEER) database (1973-2010). J Surg Res 2014;2(186):681.

[5] Kruse EJ. Palliation in pancreatic cancer. Surg Clin North Am 2010;90(2):355-64.
[6] van der Gaag NA, Kloek JJ, de Castro SMM, et al. Preoperative biliary drainage in patients with obstructive jaundice: history and current status. J Gastrointest Surg 2009;13(4):814-20.

[7] Barkin JS, Goldstein JA. Diagnostic and therapeutic approach to pancreatic cancer. Biomed Pharmacother 2000;54(7):400-9.

[8] Nagorney DM, Donohue JH, Farnell MB, et al. Outcomes after curative resections of cholangiocarcinoma. Arch Surg 1993;128(8):871-9.

[9] Nakeeb A, Pitt HA, Sohn TA, et al. Cholangiocarcinoma a spectrum of intrahepatic, perihilar and distal tumors. Ann Surg 1996;224(4):463-73.

[10] Mutignani M, Tringali A, Shah SG, et al. Combined endoscopic stent insertion in malignant biliary and duodenal obstruction. Endoscopy 2007;39(5):440-7.

[11] Ballinger AB, McHugh M, Catnach SM, et al. Symptom relief and quality of life after stenting for malignant bile duct obstruction. Gut 1994;35(4):467-70.

[12] Conroy T, Desseigne F, Ychou M, et al. FOLFIRINOX versus gemcitabine for metastatic pancreatic cancer. N Engl J Med 2011;364(19):1817-25.

[13] Robson PC, Heffernan N, Gonen M, et al. Prospective study of outcomes after percutaneous biliary drainage for malignant biliary obstruction. Ann Surg Oncol 2010;17(9):2303-11.

[14] Boulay BR, Gardner TB, Gordon SR. Occlusion rate and complications of plastic biliary stent placement in patients undergoing neoadjuvant chemoradiotherapy for pancreatic cancer with malignant biliary obstruction. J Clin Gastroenterol 2010;44(6):452-5.

[15] Dumonceau JM, Delhaye M, Tringali A, et al. Endoscopic treatment of chronic pancreatitis: European Society of Gastrointestinal Endoscopy (ESGE) clinical guideline. Endoscopy 2012;44(8):784-800.

[16] Sarkaria S, Lee HS, Gaidhane M, et al. Advances in endoscopic ultrasound-guided biliary drainage: a comprehensive review. Gut Liver 2013;7(2):129-36.

[17] Boulay BR, Parepally M. Managing malignant biliary obstruction in pancreas cancer: choosing the appropriate strategy. World J Gastroenterol 2014;20(28):9345-53.

[18] Cress RD, Yin D, Clarke L, et al. Survival among patients with adenocarcinoma of the pancreas: a population-based study (United States). Cancer Causes Control 2006;17(4):403-9.

[19] Diener MK, Heukaeufer C, Schwarzer G, et al. Pancreaticoduodenectomy (classic Whipple) versus pylorus-preserving pancreaticoduodenectomy (pp Whipple) for surgical treatment of periampullary and pancreatic carcinoma. Cochrane Database Syst Rev 2008;2:CD006053.

[20] Gouma DJ, Busch ORC, van Gulik TM. Pancreatic carcinoma: palliative surgical and endoscopic treatment. HPB (Oxford) 2006;8(5):369-76. 